\title{
Design of a Dual-Band On-Body Antenna for a Wireless Body Area Network Repeater System
}

\author{
Kyeol Kwon, Jaegeun Ha, Soonyong Lee, and Jaehoon Choi \\ Department of Electronics and Communications Engineering, Hanyang University, 17 Haengdang-dong, Seongdong-gu, \\ Seoul 133-791, Republic of Korea \\ Correspondence should be addressed to Jaehoon Choi, choijh@hanyang.ac.kr
}

Received 17 July 2012; Accepted 28 September 2012

Academic Editor: Z. N. Chen

Copyright ( $\odot 2012$ Kyeol Kwon et al. This is an open access article distributed under the Creative Commons Attribution License, which permits unrestricted use, distribution, and reproduction in any medium, provided the original work is properly cited.

A dual-band on-body antenna for a wireless body area network repeater system is proposed. The designed dual-band antenna has the maximum radiation directed toward the inside of the human body in the medical implantable communication service (MICS) band in order to collect vital information from the human body and directed toward the outside in the industrial, scientific, and medical (ISM) band to transmit that information to a monitoring system. In addition, the return loss property of the antenna is insensitive to human body effects by utilizing the epsilon negative zeroth-order resonance property.

\section{Introduction}

Recently, there has been increasing interest in wireless body area network (WBAN) systems for a variety of applications such as biomedical, military, and commercial services. Especially in biomedical applications, in order to monitor a patient's health status, an implanted device needs to collect various physiological data and wirelessly transmit the information to external medical devices in real time [1].

However, they have a short transmission range due to the low radiation efficiency and the effective radiated power (ERP) regulation of $25 \mu \mathrm{W}$. This limitation demonstrates the necessity of a dual-band on-body repeater antenna to deliver weak signals from implanted devices to external devices. In addition, antenna performance is significantly affected by body tissues due to the high dielectric constant and conductivity at the microwave frequency band. Also, the input impedance and resonance frequency cannot be changed, but the gain and radiation efficiency of an antenna can also be deteriorated when an antenna is operated on or in a body. In order to be insensitive to the human body effect, compact zeroth-order resonance (ZOR) antennas for implantable and wearable WBAN systems were proposed in $[2,3]$. Additionally, to protect the human body from radio wave exposure, the structure of the antennas for WBAN must have a low specific absorption rate (SAR) [4].
In this paper, we present a novel on-body antenna for a WBAN repeater system. The proposed antenna has a dual-band property that covers the industrial, scientific, and medical (ISM) bands and the medical implantable communication service (MICS) band. Also, the return loss of the antenna is insensitive to human body effects, and the radiation pattern of the antenna is suitable for a repeater system. The antenna structure is designed and analyzed using FEM-based commercial software ANSYS HFSS v.14.0.0 [5]. A semisolid human body phantom is used for verification of the performance of the antenna both numerically and experimentally.

\section{Antenna Design}

Figures 1(a) and 1(b) show the layout of the proposed antenna for a WBAN repeater system. The proposed antenna has the dimensions of $40 \mathrm{~mm} \times 40 \mathrm{~mm} \times 1.6 \mathrm{~mm}$, and a FR-4 dielectric with a relative permittivity of 4.4 is used as a substrate.

The antenna is comprised of a top patch fed by a microstrip line and a bottom patch fed through via connecting top and bottom patches. The top patch is used to suppress the radiation towards the outside of the body in the MICS band and transmit signals to external devices in the ISM band. 


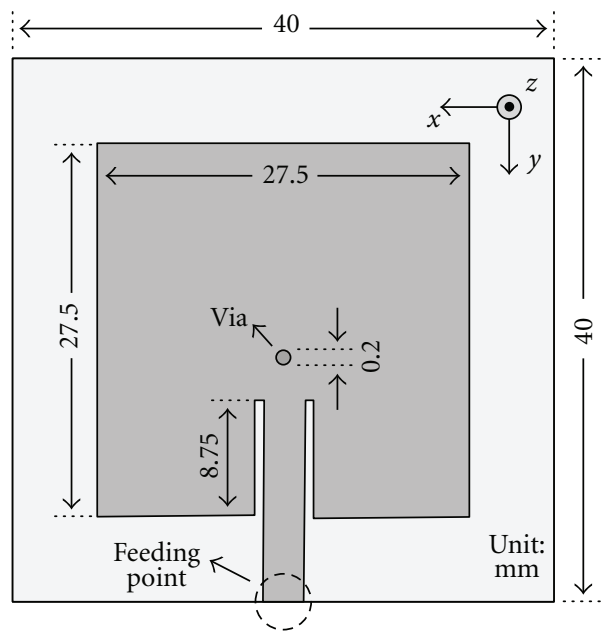

(a)

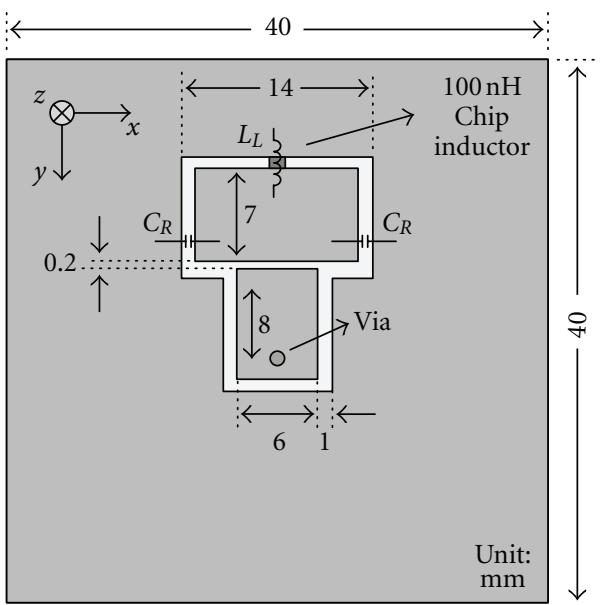

(b)
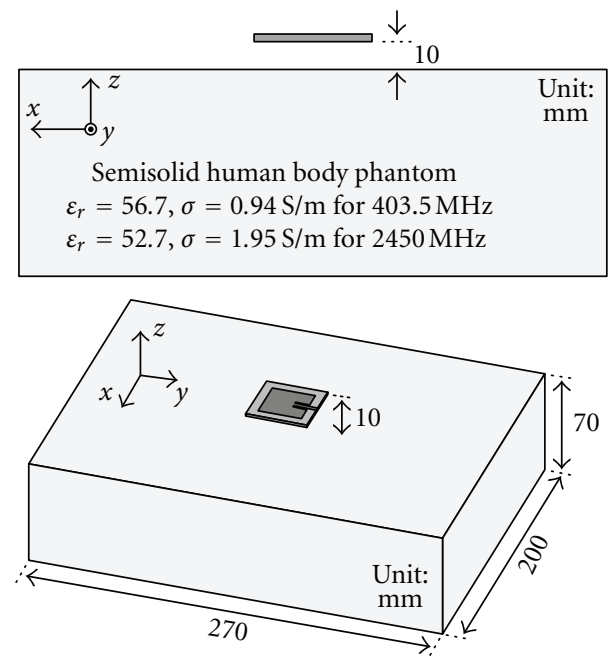

(c)

FIGURE 1: Configuration of the proposed antenna: (a) top view, (b) bottom view, (c) calculation setup for the on-body antenna on a semisolid human body phantom.

The bottom patch is designed to communicate with the implanted devices in the MICS band and to reduce the human body effects of the ISM band. The bottom patch is fed by using a via at the center of the top patch, and a chip inductor with a value of $100 \mathrm{nH}$ is mounted between the bottom patch and the ground plane to realize epsilon negative (ENG) ZOR. The gap between the bottom patch and the ground plane can be modeled as the shunt capacitance $\left(C_{R}\right)$, and the chip inductor can be modeled as a shunt inductor $\left(L_{L}\right)$. The ZOR frequency from the above circuit description can be found using:

$$
\omega_{0}=\frac{1}{2 \pi \sqrt{C_{R} \times L_{L}}},
$$

where $\omega_{0}$ is the ZOR frequency [6]. By adopting the large inductance of the chip component, the bottom patch can still have a compact size $\left(0.019 \lambda_{0} \times 0.0094 \lambda_{0}\right)$ that is even smaller than the top patch. This allows a reduction in the off-body side radiation in the MICS band.
For a numerical simulation of the antenna, a homogeneous semisolid human body phantom $(200 \mathrm{~mm} \times$ $270 \mathrm{~mm} \times 70 \mathrm{~mm}$ ) is modeled as illustrated in Figure 1(c).

\section{Results and Discussion}

Figure 2 shows the fabricated antenna and the measurement setup in an anechoic chamber. To measure the return loss and radiation pattern, the antenna was placed $10 \mathrm{~mm}$ away from the surface of the fabricated semisolid phantom, and a Styrofoam slab $\left(\varepsilon_{r}=1.0\right)$ was inserted between the antenna, and the phantom for spacing.

In Figures 3(a) and 3(b), the measured electrical properties of the fabricated phantom using an Agilent 85070E Dielectric Probe Kit and 8719ES network analyzer together are shown. As shown in the figure, the measured relative dielectric constant and conductivity of the phantom $\left(\varepsilon_{r}=\right.$ 56.1 and $\sigma=0.92 \mathrm{~S} / \mathrm{m}$ at $403.5 \mathrm{MHz}$, and $\varepsilon_{r}=52.5$ and $\sigma=1.98 \mathrm{~S} / \mathrm{m}$ at $2450 \mathrm{MHz}$ ) closely agreed with the values 


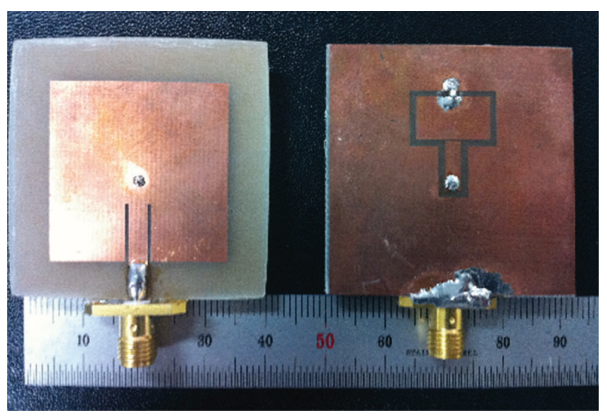

(a)

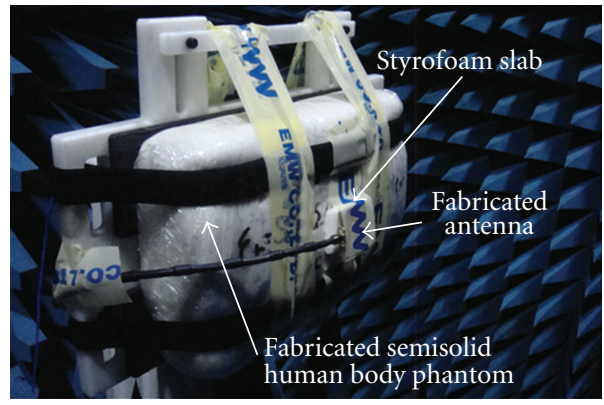

(b)

Figure 2: Photographs of the manufactured antenna and measurement setup: (a) manufactured antenna, (b) Measurement setup.

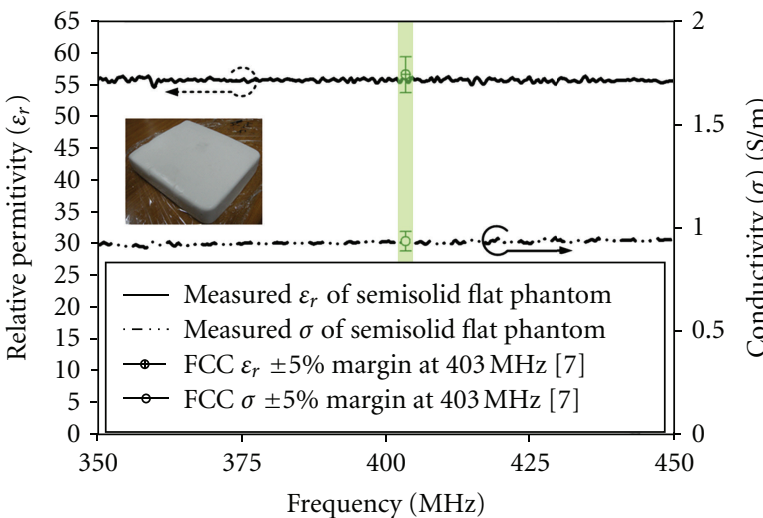

(a)

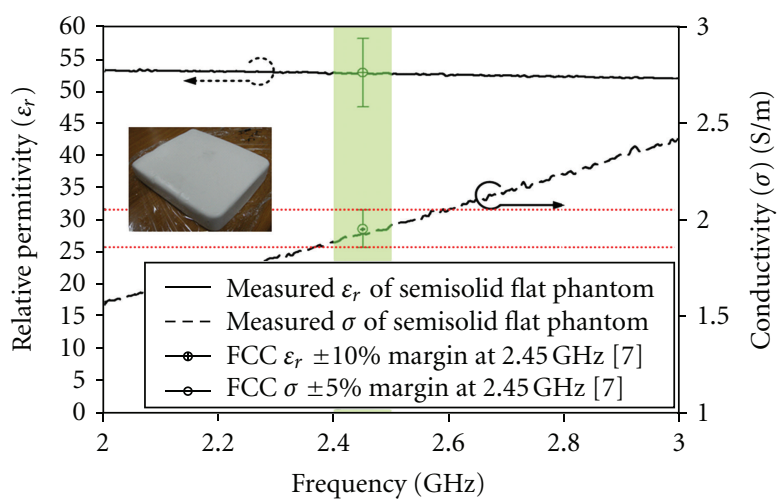

(b)

FIGURE 3: Measured 3D radiation pattern of the antenna on a semisolid human body phantom: (a) at $403.5 \mathrm{MHz}$, (b) at $2450 \mathrm{MHz}$.

from the equivalent electrical properties $\left(\varepsilon_{r}=56.7\right.$ and $\sigma=0.94 \mathrm{~S} / \mathrm{m}$ at $450 \mathrm{MHz}$, and $\varepsilon_{r}=52.7$ and $\sigma=1.95 \mathrm{~S} / \mathrm{m}$ at $2450 \mathrm{MHz}$ ) of the whole human body [7].

Figure 4 shows the return loss characteristics of the proposed antenna in free space and on the human body phantom. All the setup of the measurements and the calculations had been taken for a 50 -ohm load. The measured results agreed well with the calculated results. The measured $10 \mathrm{~dB}$ impedance bandwidths of the antenna were $3.7 \%(396-411 \mathrm{MHz})$ at the MICS band and 3.7\% (2398$2489 \mathrm{MHz}$ ) at the ISM band. In addition, the return loss property of the proposed antenna was insensitive to the existence of the human body phantom. This is because the ZOR frequency at the MICS band is insensitive to the surrounding medium [2], and the bottom patch reduces the human body effect in the ISM band.

Figures 5(a) and 5(b) depict the simulated electric field distributions of the substrate at each resonance frequency of the proposed antenna. In Figure 5(a), the electric field distribution is in phase by the virtue of the ENG ZOR resonance emanating from the bottom patch at $403.5 \mathrm{MHz}$. Besides, typical $180^{\circ}$ out of phase property, which is a general characteristic of a patch antenna, is observed in Figure 5(b) since the resonance of the top patch occurs at $2450 \mathrm{MHz}$.

Figures 6(a) and 6(b) depict the measured radiation patterns of the antenna on a semisolid human body phantom. Even though the ZOR antenna has a dipolar radiation

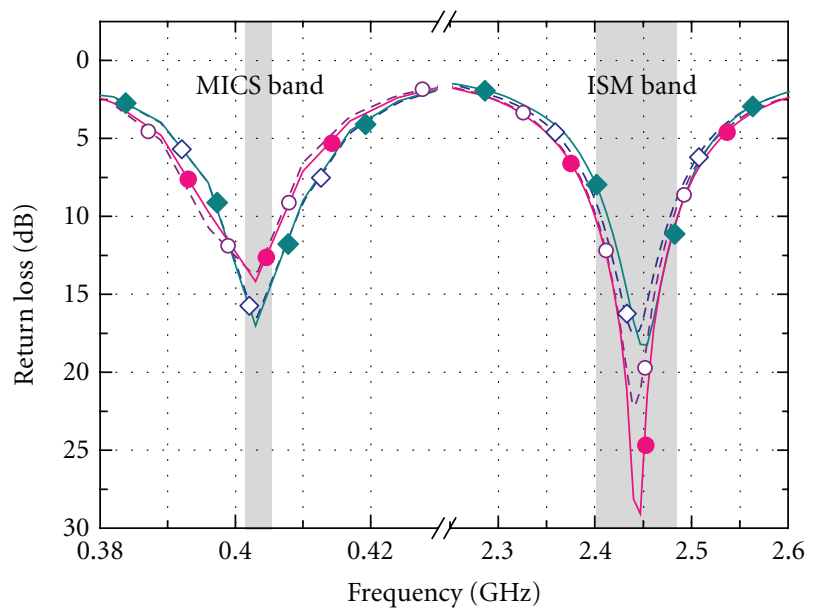

$-\diamond-$ Calculated RL w/o phantom

- - Calculated RL w/ phantom

$\neg$ Measured RL w/o phantom

$\rightarrow$ Measured RL w/ phantom

FIGURE 4: Return loss characteristics of the proposed antenna.

pattern, the maximum power was delivered toward the body at the MICS band due to the suppression caused by the top patch; therefore, the proposed repeater antenna is advantageous for communication with other implanted devices in the MICS band. In the ISM band, on the other hand, the maximum power was delivered outward from 


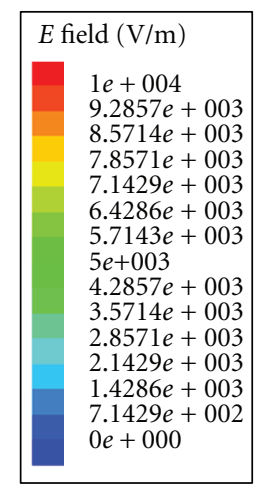

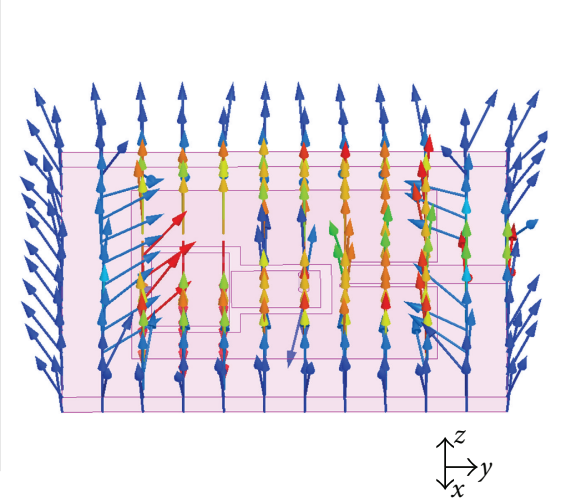

(a)
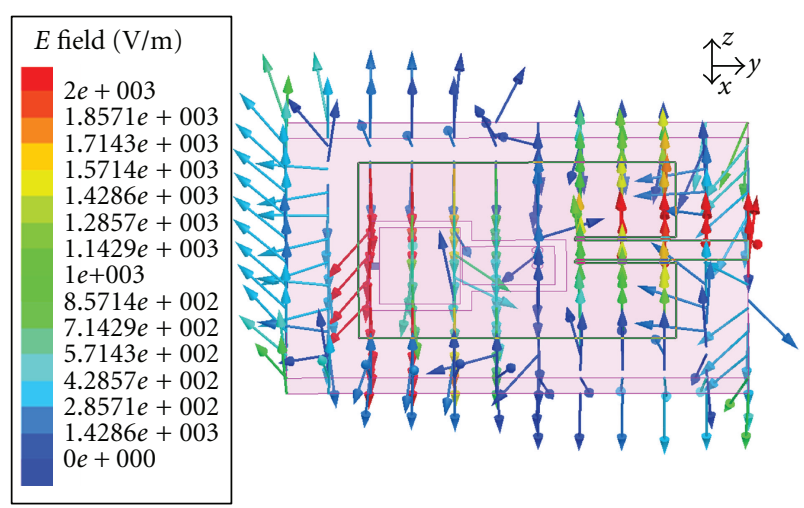

(b)

FIGURE 5: Simulated electric field distributions of the proposed antenna: (a) for the MICS band (403.5 MHz), (b) for the ISM band $(2450 \mathrm{MHz})$.
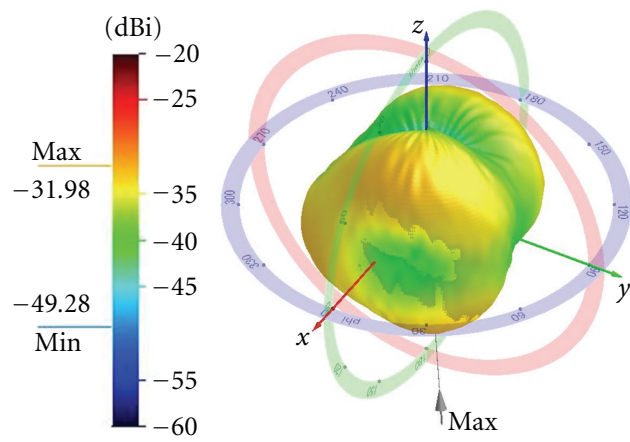

(a)

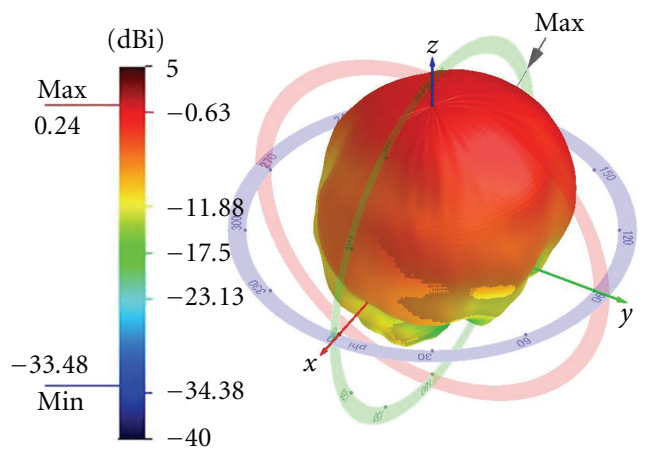

(b)

FIGURE 6: Measured 3D radiation patterns of the antenna on a semisolid human body phantom: (a) at $403.5 \mathrm{MHz}$, (b) at $2450 \mathrm{MHz}$.

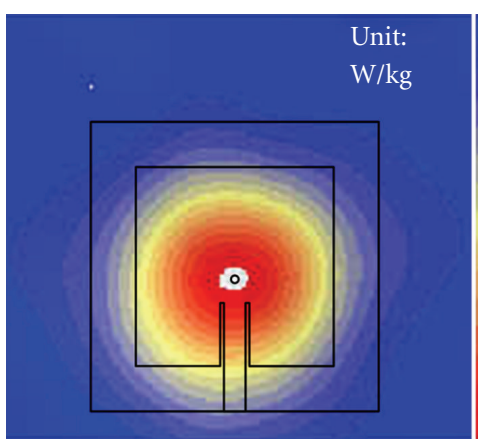

(a)

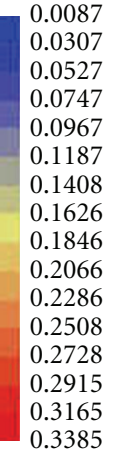

0.3385

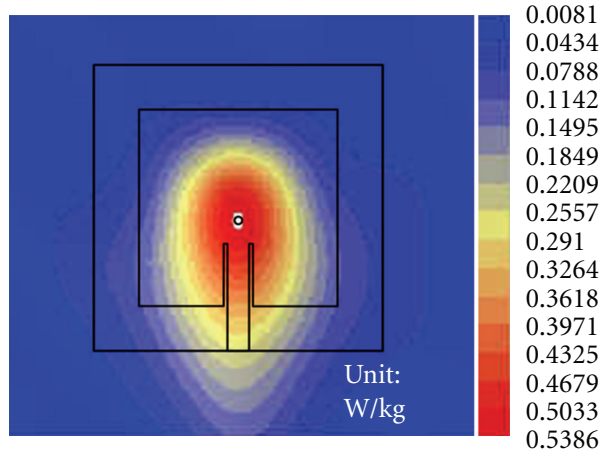

(b)

Figure 7: Measured SAR distribution: (a) at $403.5 \mathrm{MHz}$, (b) at $2450 \mathrm{MHz}$.

the body, which improves the communication efficiency between the repeater and an external device. In addition, the peak gains were $-31.98 \mathrm{dBi}$ and $0.24 \mathrm{dBi}$ at $403.5 \mathrm{MHz}$ and $2450 \mathrm{MHz}$, respectively.

The measured SAR distributions are shown in Figure 7. The SAR was measured at the Radio Research Agency of Korea using the ESSAY system [8]. The proposed antenna is excited by the signal generator. The input power of $250 \mathrm{~mW}$, which is usually used for SAR measurements of mobile application devices, is used to measure SAR. Hot spots are observed underneath the center of the top patch where the via is located, and the maximum SAR values were $0.411 \mathrm{~W} / \mathrm{kg}$ at $403.5 \mathrm{MHz}$ and $0.455 \mathrm{~W} / \mathrm{kg}$ at $2450 \mathrm{MHz}$. Although a high input power of $250 \mathrm{~mW}$ was delivered to the antenna, the maximum values of the measured SAR were still well below the regulated SAR limitation $(1.6 \mathrm{~W} / \mathrm{kg})$ of the American 
National Standards Institute (ANSI/IEEE) for short-distance biotelemetry [9].

\section{Conclusion}

We proposed a dual-band on-body antenna for the WBAN repeater system. The bandwidths of the proposed antenna were wide enough to cover the MICS band (402-405 MHz) and ISM band (2400-2485 MHz). Also, the resonance frequencies are stationary whether the antenna is placed in the air or on a human body phantom. In addition, the radiation pattern of the antenna is advantageous for communication with implant devices in the MICS band and external devices in the ISM band. The measured maximum SAR values were low enough to conform to the SAR limitation of the ANSI. Consequently, the proposed antenna can be a good candidate for a WBAN repeater system owing to the dualband property, the insensitivity to any human body effect, and the desirable radiation pattern.

\section{Acknowledgments}

This work was supported by the National Research Foundation of Korea (NRF) Grant funded by the Korean government (MEST) (no. 2012-0005655).

\section{References}

[1] P. S. Hall and Y. Hao, Antennas and Propagation for Body-Centric Wireless Communications, Artech House, Norwood, Mass, USA, 2006.

[2] J. Ha, K. Kwon, and J. Choi, "Compact zeroth-order resonant antenna for implantable biomedical service applications," Electronics Letters, vol. 47, no. 23, pp. 1267-1269, 2011.

[3] J. Lee, S. I. Kwak, and S. Lim, "Wrist-wearable zeroth-order resonant antenna for wireless body area network applications," Electronics Letters, vol. 47, no. 7, pp. 431-433, 2011.

[4] U. Kim and J. Choi, "Design of a microstrip patch antenna with enhanced F/B for WBAN applications," IEICE Transactions on Communications, vol. E94-B, no. 5, pp. 1135-1141, 2011.

[5] HFSS: High Frequency Structure Simulator Based on the Finite Element Method, v.14.0.0, ANSYS.

[6] A. Lai, C. Caloz, and T. Itoh, "Composite right/left-handed transmission line metamaterials," IEEE Microwave Magazine, vol. 5, no. 3, pp. 34-50, 2004.

[7] D. L. Means and W. Kwok, Evaluating Compliance with FCC Guidelines for Human Exposure to Radiofrequency Electromagnetic Fields, Supplement C (edition 01-01) to OET Bulletin 65 (Edition 97-01), Federal Communications Commission Office of Engineering \& Technology, June 2001.

[8] http://emfsafety.koreasme.com/.

[9] "IEEE Standard for Safety Levels with Respect to Human Exposure," IEEE Standard C95. 1-1999, 1999. 

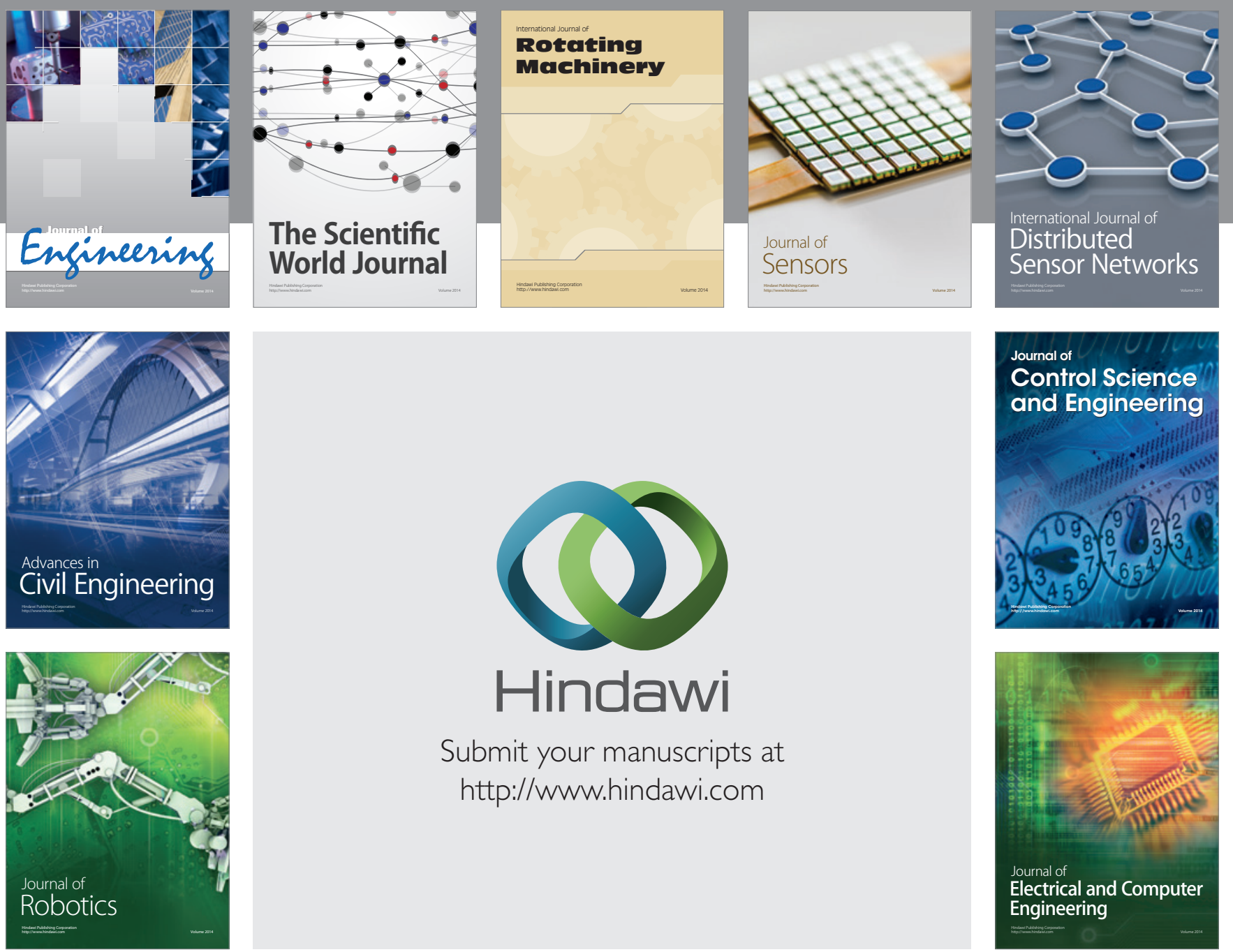

Submit your manuscripts at

http://www.hindawi.com
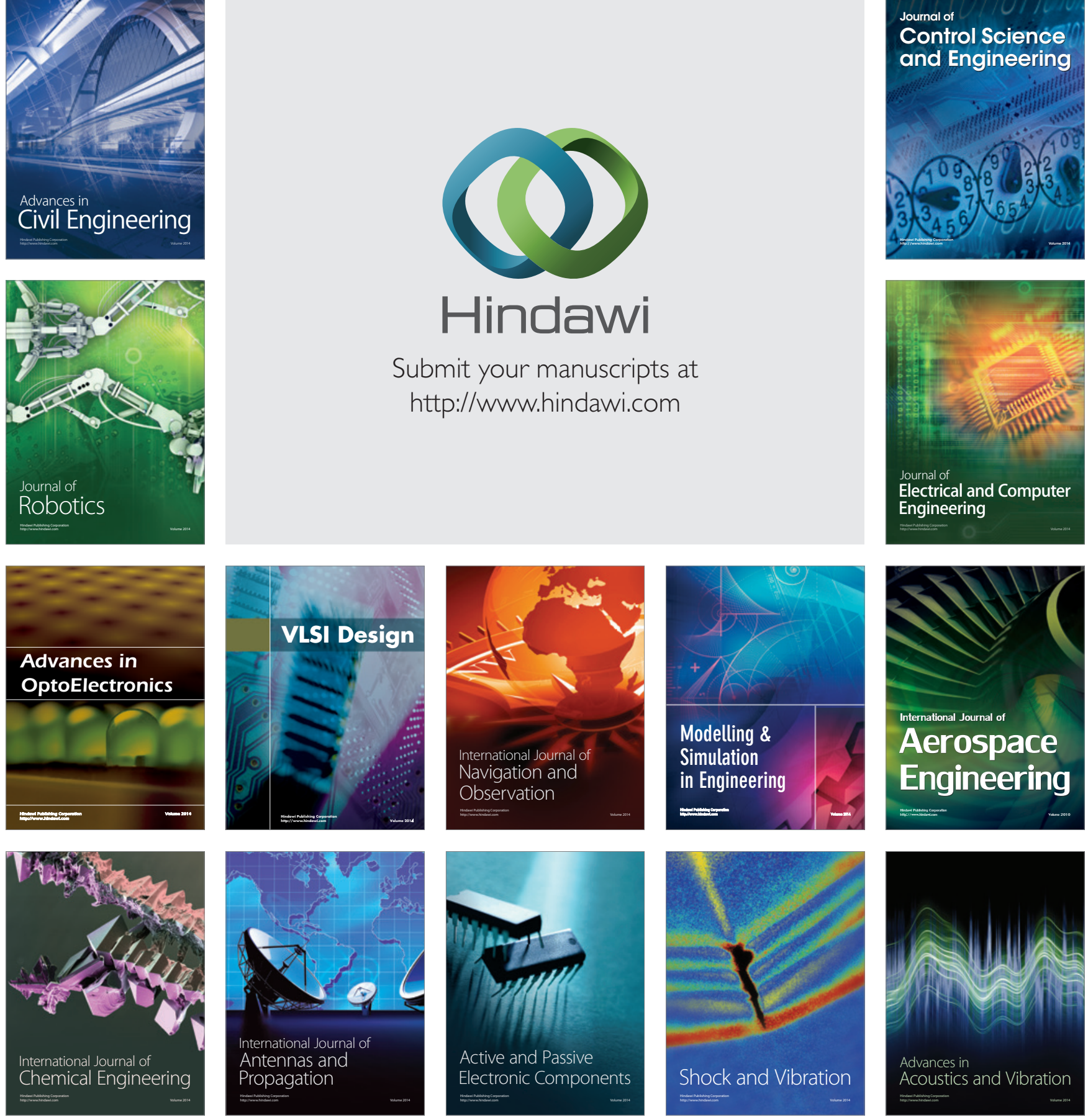